\title{
GCMPR: Gateway-Centric Multi-path Routing for Internet Connectivity of Wireless Mobile Ad Hoc Network
}

\author{
Yongqiang Liu, Wei Yan, and Yafei Dai \\ Computer Networks and Distributed Systems Laboratory, Peking University, \\ Room 1716, Science Building No.1, Peking University, Beijing, China \\ \{lyq, yanwei, dyf\}@net.pku.edu.cn
}

\begin{abstract}
Connecting Mobile Ad Hoc Network (MANET) to the Internet will extend Internet into a new territory by making web service available "anytime, anywhere". However, many simulations and practical experiments have shown that when communication is between mobile nodes in MANET and fixed nodes on Internet, the bandwidth is asymmetrical and the available throughput is greatly limited. In this paper, we present a gateway-centric multi-path routing protocol (GCMPR), which ensures every mobile node has a set of link-disjoint and loop-free paths to gateway. The novel routing algorithm over multiple paths can provide higher aggregate bandwidth and improve the end-to-end delay. Simulations for comparing performance between GCMPR and other connectivity methods show that GCMPR achieves $40 \%$ throughput enhancement and remarkable improvement for the end-to-end delay. Furthermore, GCMPR can be easily applied to other scenarios in MANET when some hosts provide special service and are visited frequently.
\end{abstract}

\section{Introduction}

Mobile ad-hoc communication [1] has been one of the most active research areas in the past decade. For many applications in the mobile environment, it is much desired that a self-organizing ad hoc network is somehow connected to the world-wide Internet. We refer to such connecting networks as wireless hybrid networks. Usually, a MANET node with Internet access can operate as a gateway and provide Internet access to other nodes in the MANET.

There have been some researches on connectivity for MANET, such as node addressing[2], gateway discovery[3] and routing algorithms[4-5]. The performance evaluations of wireless hybrid networks [6,7] have shown that the communication bandwidth between MANET nodes and Internet nodes is asymmetrical. As illustrated in Fig.1-(a), the end-to-end throughput is actually determined by the bandwidth between MANET nodes and the gateway. The aim of our work is to develop a multipath routing protocol for providing higher aggregate bandwidth to gateway and improve the performance of the wireless hybrid network. 


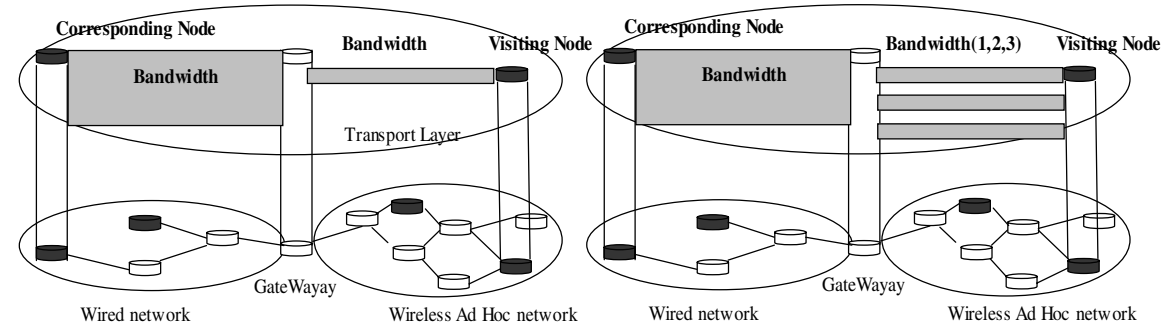

Fig. 1. Bandwidth illustration over wireless hybrid network

Some multi-path routing approaches have been proposed, such as TORA[8],SMR[9], AOMDV[10], and AODVM[11]. But none of them can be directly applied to the wireless hybrid network, because these protocols require the destination node to be in MANET. To solve this problem, we present a GatewayCentric Multi-Path Routing (GCMPR) protocol. As illustrated in Fig.1-(b), GCMPR is a hybrid routing protocol. The gateway pushes proactively its link-disjoint routing entries into the routing tables of the rest of the nodes in MANET. The communications among MANET nodes still use single path routing on-demand.

The advantages of GCMPR can be summarized as follows:

1. When there is a large percentage of traffic between MANET nodes and Internet nodes traversing the gateway, GCMPR can greatly improve the end-to-end throughput compared to the single path routing approach.

2. When there are many nodes need to establish multi-path routing to the gateway, GCMPR requires fewer additional control messages than other multi-path routing protocols, therefore, lower overhead in MANET.

3. In other routing approaches, only the source node has multi-path information to the destination. The intermediate nodes in GCMPR also keep multi-path information to the gateway. Therefore, GCMPR is more effective in recovering the broken path.

The remainder of this paper is organized as follows. Session 2 describes the protocol mechanism in detail. Performance evaluation by simulation is presented in Section 3 and concluding remarks are given in Section 4.

\section{Gateway-Centric Multi-path Routing in Hybrid Network}

\subsection{Protocol Overview}

The GCMPR is a distributed message-based routing scheme. As illustrated in Fig.2, the basic idea of message-based routing is that a gateway information message is generated by the gateway and sent to its neighbors. The neighbors add their own information into the message and forward it to the downstream nodes. Analyzing the messages coming from different neighbors, the MANAT node can form multiple linkdisjoint paths to the gateway. The main steps of GCMPR are as follows: 


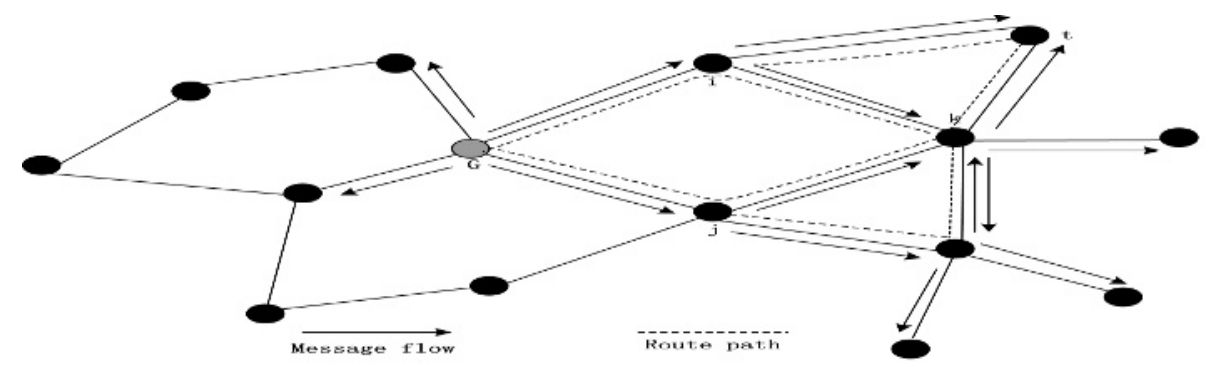

Fig. 2. An example of setting up multiple paths to the gateway with GCMPR

1. Gateway broadcasts periodically a gateway advertisement (GWADV) message throughout the ad hoc network.

2. When the GWADV message is received from its neighbors, a node makes a decision based on its states. These states include following scenarios: 1) whether the GWADV can provide the information for it to find another link-disjoint path to the gateway. 2) whether the message should be forwarded to its downstream nodes. To achieve these two goals, each MANET node uses a variant PID to identify each path to the gateway. The PID is defined as follows:

$$
P I D=<n e x t_{-} \text {hop, first_hop }>
$$

where the next_hop is the IP address of the upstream neighbor along the path and first_hop is the IP address of the first hop just before the gateway. For the path1 $(\mathrm{t}->\mathrm{k}-$ $>\mathrm{j}->\mathrm{G})$ in Fig2, from the view of node $\mathrm{t}, P I D_{1}=<k, j>$

We define that

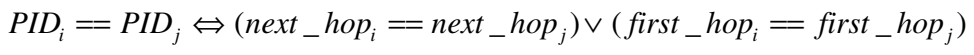

$$
\begin{aligned}
& P I D_{i} \neq P I D_{j} \Leftrightarrow\left(\text { next_hop }_{i} \neq n e x t_{-} \text {hop }_{j}\right) \wedge\left(\text { first_hop }_{i} \neq \text { first_hop }_{j}\right)
\end{aligned}
$$

By comparing the PIDs in the GWADV messages, GCMPR can guarantee that the paths to the gateway are link-disjoint and loop-free. This is accomplished by applying GWADV forwarding rule to each intermediate node. Details and proof of the forwarding rule will be discussed in following sessions.

3. In order to reduce the overhead in the ad hoc network, only useful GWADV messages which can create a new link-disjoint path or shorter path to downstream nodes are modified and forwarded by the receiving node.

4. When a node detects its next hop in the path out of its range, it performs a micro repair to find an alternative path to the gateway and the cost of this repair is very low.

5. If a mobile node (S) wants to communicate with another node (D), S estimates the location of D (whether D is in MANET or on Internet) by broadcasting a networkwide RREQ and waiting for corresponding RREP. In GCMPR, RREQ is extended to contain additional information for forming reverse multi-path routing to the source. 


\subsection{Gateway Advertisement Message and Gateway Routing Table}

A gateway can advertise its presence by sending a GWADV message. The GWADV message contains information for propagating a new link-disjoint path to the gateway for downstream nodes. The general format of this message is shown in Fig.3, where ADVERTISEMENT ID (ADV_ID) is the sequence number of the message and lager ADV_ID means fresher message.

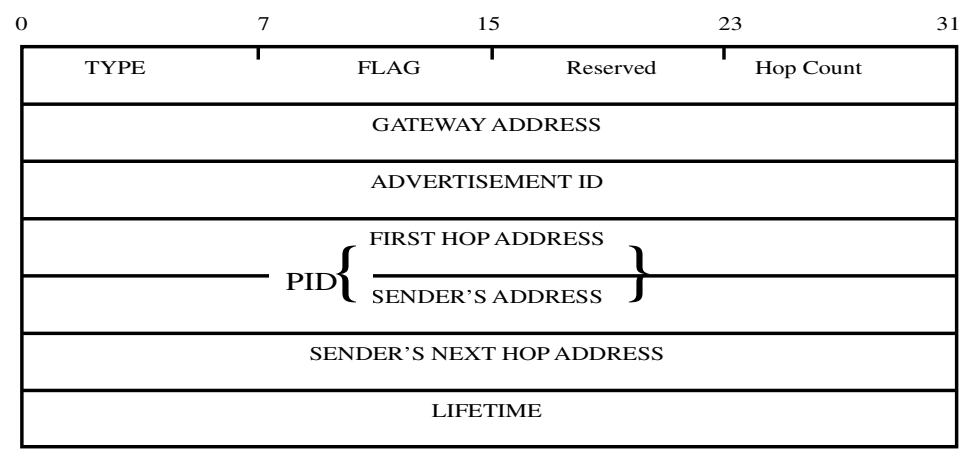

Fig. 3. Format of Gateway Advertisement Message

As introduced in section1, in GCMPR, each MANET node only establishes multipath routing to the gateway and keeps single path routing among MANET nodes. An additional gateway routing table (called gw_rtable) is designed to record path information to the gateway. The structure of the gw_rtable and the relationship between the two routing tables are illustrated in Fig.4.

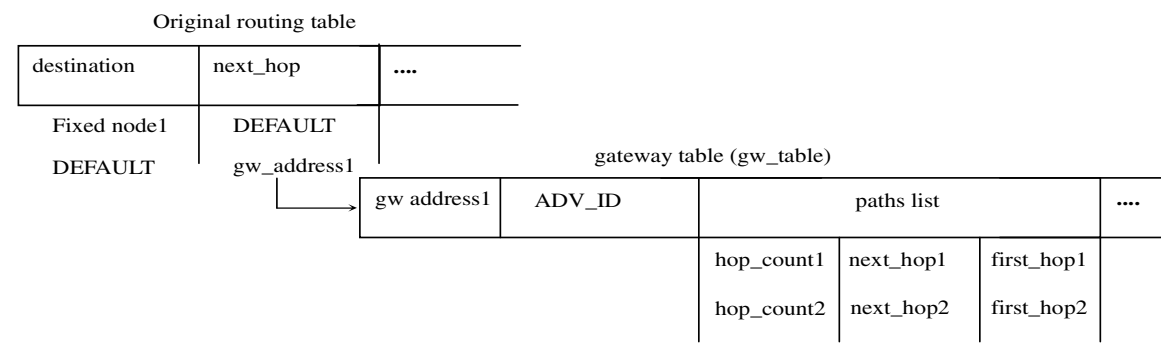

Fig. 4. The structure of gw_rtable and the relationship between the two routing tables

\subsection{GWADV Forwarding Rule}

As mentioned in section 2.1, GCMPR uses GWADV forwarding rule to form multiple loop-free paths to the gateway. Moreover, the rule can reduce additional routing overhead and prevent downstream nodes from receiving wrong PID information. The detailed GWADV forwarding rule is shown in Fig.7. 


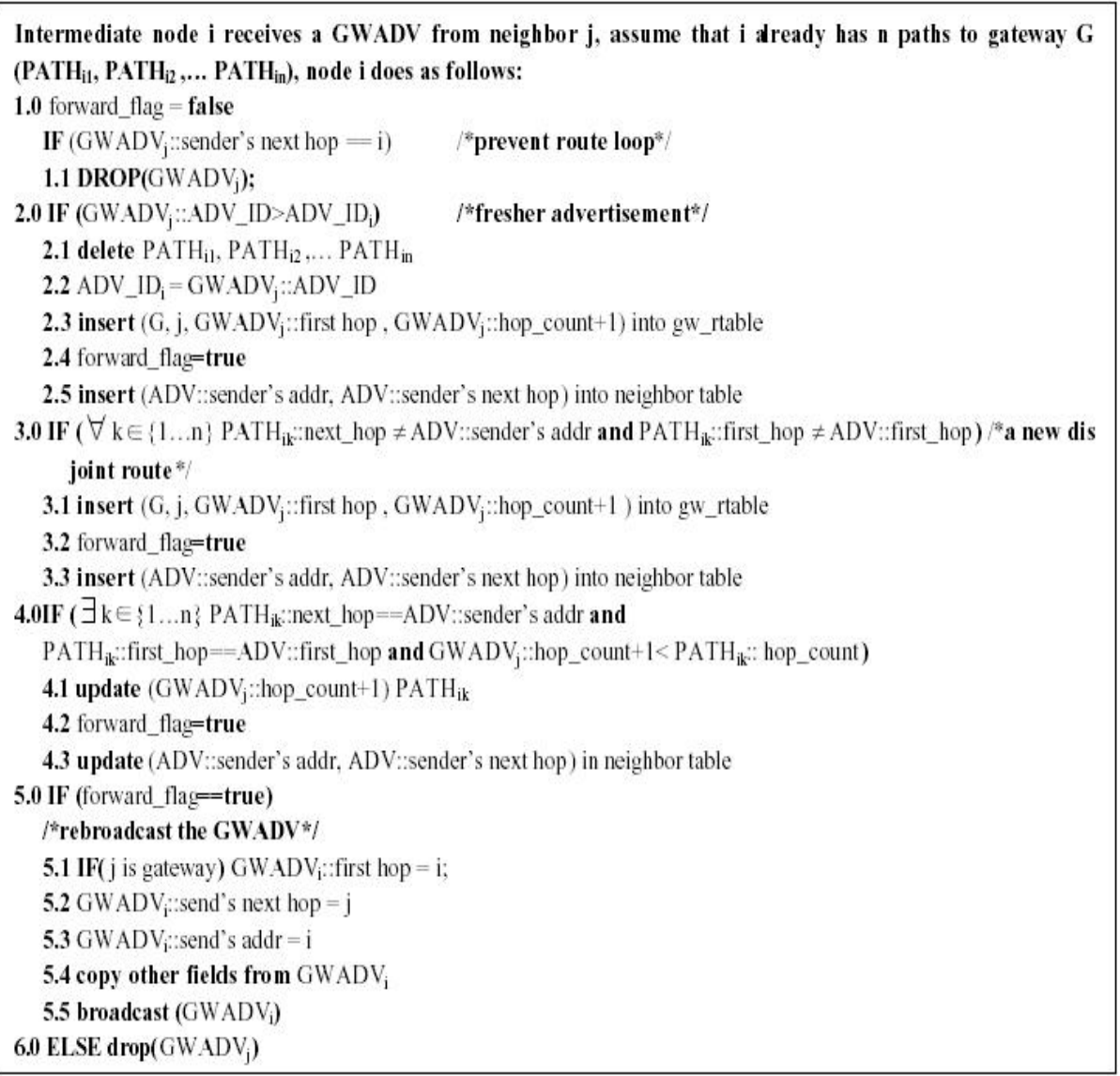

Fig. 5. GCMPR GWADV forwarding rule

\section{Performance Evaluation}

\subsection{Simulation Environment}

The simulations were done with ns2 [12]. All of the simulations were run in an ad hoc network consisting of 50 nodes uniformly spreading in an 1000x1000 meter area. Nodes are equipped with an IEEE 802.11 radio network interface, operating at $1 \mathrm{Mbps}$ with $250 \mathrm{~m}$ transmission range. Nodes move according to the Random Waypoint mobility model, at uniformly distributed speed between 0 and $15 \mathrm{~m} / \mathrm{s}$. The traffic pattern consists of several CBR/UDP connections between randomly chosen source and two fixed nodes in wired network. The sending interval at the source is $20 \mathrm{~ms}$ and packet size is 80 bytes.

In [11], the AODV protocol is modified to implement solutions to the wireless hybrid network by adding node addressing and the gateway discovery methods. We 
refer to the modified AODV as AODV+. There are two gateway discovery mechanisms In [11], the AODV protocol is modified to implement solutions to the wireless hybrid network by adding node addressing and the gateway discovery methods. We refer to the modified AODV as AODV+. There are two gateway discovery mechanisms in AODV+: reactive gateway discovery and proactive gateway discovery. In the simulation, four protocols are measured. They are AODV+ with reactive gateway discovery (called reactive AODV+), AODV+ with proactive gateway discovery (proactive AODV+), AODV incorporating GCMPR without micro repair (GCMPR) and AODV+GCMPR with micro repair (GCMPR with micro repair). We use salvage mode as multi-path selection scheme [10] and the gateway broadcasts an advertisement in every 5 seconds.

Three important metrics of protocol performance are evaluated:

Packet Throughput: This is the product of the size of the packet and the number of packets received by destination in a unit time. Data packets can be dropped in the network either at the source or at intermediate nodes for node mobility and wireless channel collision.

End-to-End Delay of Data Packets: This includes all possible delays caused by buffering during route discovery, queuing delay at the interface, retransmission delays at the MAC, propagation time and transfer time.

Routing Control Messages Overhead: Routing control messages include gateway discovery advertisement, request and reply. The metric is the ratio of the number of control message to the total data packets received by the destination.

\subsection{Simulation Results}

Fig.6, 7 and 8 show the results with varying mean node speeds. Fig 6 compares the packet throughput performance of four protocols. The throughput of all protocols decreases with increase of mean node-speed, because nodes will drop packets if they have no routes to forward when topology changes. GCMPR always drops fewer packets than other protocols and the throughput improvement is up to about $40 \%$. This is because of the availability of alternative paths to forward the packets when one path fails. The GCMPR with micro repair outperforms other protocols because of its rapid route recovery at intermediate nodes.

Fig.7 shows the end-to-end delay of four protocols. As expected, the delay increases with mean node-speed grows. This is because of the increase in the number of route failures and the consequent packet cache. GCMPR with micro repair improves the delay almost by a factor of two. So the improvement of packet end-to-end delay is more significant than that of the throughput.

In Fig.8, it can be seen that GCMPR has more control overhead (average about $20 \%$ ), because it uses additional GWADV messages to form multiple paths to the gateway. With the increasing mobility, reactive AODV+ has to send more route discovery requests to discover the path to the gateway and therefore higher overhead. 


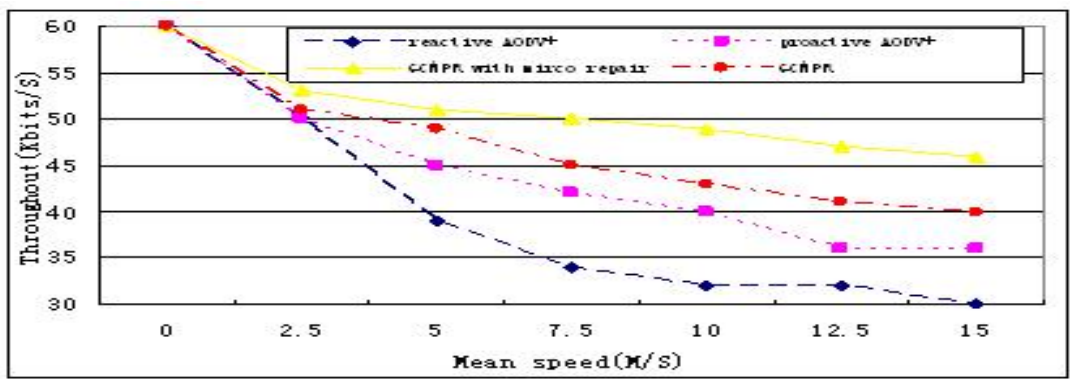

Fig. 6. Throughput with varying mobility

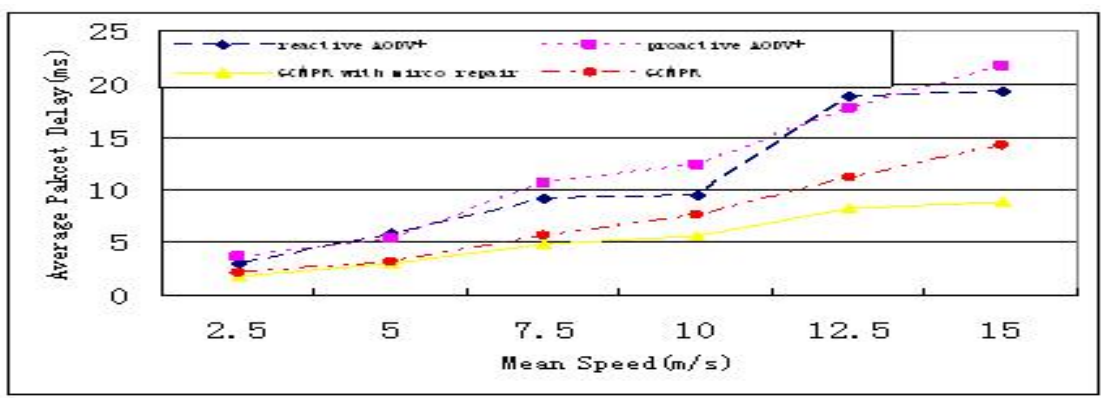

Fig. 7. End-to-end Delay with varying mobility

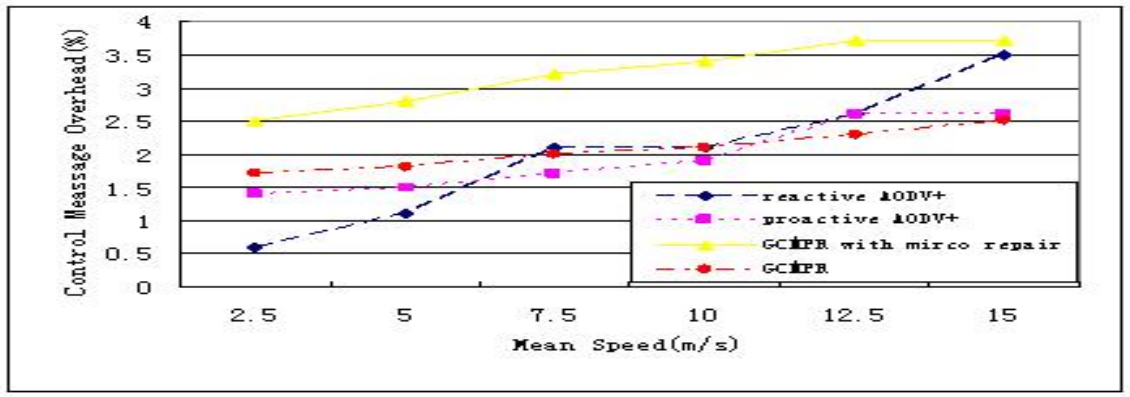

Fig. 8. Routing overhead with varying mobility

\section{Conclusions}

Routing protocols with multi-path capability can improve the bandwidth between the gateway and mobile nodes in MANET, because it can effectively deal with mobilityinduced route failures. In this paper, we have proposed a gateway-centric multi-path protocol (GCMPR) which ensures that each mobile node has a set of link-disjoint and loop-free paths to the gateway. Another feature of GCMPR is the micro repair with minimal additional overhead. We have studied the performance of GCMPR relative to 
AODV+ using ns-2 simulations under varying mobility scenarios. The results show that GCMPR can improve the throughput by up to $40 \%$ and offers a significant reduction in the end-to-end delay.

\section{Reference}

1. Alex Ali Hamidian: A Study of Internet Connectivity for Mobile Ad Hoc Networks in NS 2. Master's thesis, Lund Sweden (2003)

2. Jönsson U, Alriksson F,Larsson T: MIPMANET- Mobile IP for Mobile Ad Hoc Networks. In Proceedings of the Workshop on Mobile Ad Hoc Networking and Computing (MobiHoc), Boston, USA (2000)

3. Xi J.; Bettstetter C: Wireless Multihop Internet Access: Gateway Discovery, Routing and Addressing. In Proceedings of the International Conference on Third Generation Wireless and Beyond (3Gwireless'02), San Francisco, USA(2002)

4. Perkins C: IP Mobility for IPv4, Revised. draft-ietf-mobileip-rfc2002-bis-08.txt(2001)

5. Wakikawa R., Malinen J., Perkins C., Nilsson A: Global Connectivity for IPv6 Mobile Ad Hoc Networks, IETF Internet Draft, November 2001. Work in progress.

6. S. Xu and T. Saadawi: Does the IEEE 802.11 MAC protocol Work Well in Multihop Wireless Ad Hoc Networks. IEEE Communication Magazine, Volume 39(2001) 130-137.

7. G. Anastasi, E. Borgia, M. Conti, E. Gregori: IEEE 802.11Ad Hoc Networks: Performance Measurement. IIT Internal Report(2003)

8. V.D. Park and M.S. Corson: A Highly Adaptive Distributed Routing Algorithm for Mobile Wireless Net-works. Proceedings of IEEE INFOCOM'97 Conf (1997)

9. Lee, S.-J., Gerla, M: Split Multipath Routing with Maximally Disjoint Paths in Ad Hoc Networks. IEEE International Conference on Communications, Vol. 10 (2001)

10. Marina, M.K., Das, S.R.: On-demand Multipath Distance Vector Routing in Ad Hoc Networks. Proceedings of the International Conference for Network Protocols (2001)

11. Ye, Z., Krishnamurthy, S.V., Tripathi, S.K.: A Framework for Reliable Routing in Mobile Ad Hoc Networks. IEEE INFOCOM (2003)

12. K. Fall: The ns Manual. http://www.isi.edu/ns/nsdocumentation.htm(2002) 